\title{
Influence of liquid bridge volume on the onset of oscillation in floating zone convection II. Numerical simulation
}

\author{
Z.M. Tang, W.R. Hu * \\ Institute of Mechanics, Chinese Academy of Sciences, 15 Zhong Guan Cun Road, Beijing 100080, People's Republic of China
}

Received 29 October 1993; manuscript received in final form 11 January 1994

\begin{abstract}
The onset of oscillation in the floating zone convection driven by the gradient of surface tension was studied numerically for an unsteady and two-dimensional model, and studies were concentrated on the influence of liquid bridge volume on the onset of oscillation in comparison with the experimental results in the Paper I. The numerical results agree with the experimental ones presented in the previous paper, in which the distributions of critical applied temperature difference depending on the volume of liquid bridge and a gap range of liquid volume in marginal stability curve were obtained.
\end{abstract}

\section{Introduction}

The floating zone method is one of the important ways for crystal growth, either on the ground or in space, of materials processing, and numerical calculations have been applied successfully to simulate the process. The model of half floating zone with small typical length scale was studied experimentally in previous work [1-3], where the thermocapillary effect dominated the process. The experimental results show that a new geometrical parameter, the ratio volume of liquid bridge, is sensitive to the onset of oscillation, in addition to the typical length scale and aspect ratio adopted extensively as the usual geometrical parameters. The previous work of Paper I gave in detail the

\footnotetext{
${ }^{*}$ Corresponding author.
}

distribution of the critical applied temperature difference $(\Delta T)_{\mathrm{c}}$ depending on the volume ratio of liquid bridge with a break at a certain range of volume ratio, and the theoretical implication on the instability in the thermocapillary convection of half floating zone was discussed. The experimental result of marginal stability curve is important for understanding the features of thermocapillary oscillatory convection and the applications on materials processing. In the present paper, the same problem is studied by numerical approach, and the results are compared with the experimental ones in explanation of the oscillatory mechanism.

A hybrid method of fractional steps has been successfully applied to the unsteady and two-dimensional problem of half floating zone convection [4,5], and this numerical approach is used to study the influence of liquid bridge volume on the 
critical applied temperature difference. The experimental problem should be described by the three-dimensional model in a real case; however, the onset of oscillation could be simplified at the first step as a two-dimensional model in the view point of mechanism studies. It seems that the two-dimensional model will not change the essential features, although there may have been quantitative changes between two-dimensional and three-dimensional model.

The numerical results of the present paper agree quantitatively with the experimental results presented in Paper I. In the next section, the physical model and its mathematical description are given based on the Boussinesq approximation. Numerical simulation method and the results are presented in Section 3, and the conclusion is given in the last section.

\section{Mathematical description}

The two-dimensional half floating zone consists of a liquid column confined by the surface tension in the gap between two parallel planes with the same width; the upper one has relatively high temperature in comparison with the lower one. In the present paper, the width $d_{0}=4 \mathrm{~mm}$ of the liquid bridge and the aspect ratio $L / d_{0}=1$ are adopted, where $L$ is the height of the liquid bridge. Fig. 1 shows the configuration of the two-dimensional thermotemperature liquid bridgc on the ground, and the direction of gravity directed downward. The curve shape of the free surface is not changed in the calculation, for simplification. The ratio of the minimum width $d_{\min }$ to the width of the wall $d_{0}$ of the liquid bridge is adopted to describe the non-dimensional volume ratio of the liquid bridge, and $d_{\min } / d_{0}$ is changed in the range of 0.5 to 0.95 . The dynamic behaviour of the fluid in the liquid bridge is governed by incompressible continuous equation, Navier-Stokes equation and energy equation under the associated initial and boundary conditions.

The height $L$ of the liquid bridge and applied temperature difference $\Delta T^{\prime}$ between the top and bottom walls are adopted as the characteristic length and typical temperature, and the reference velocity $U_{\mathrm{r}}=\left|\partial \sigma / \partial T^{\prime}\right| \Delta T^{\prime} / \rho \nu$ is introduced due to the equilibrium between surface tension and viscous force at the free surface. The dimensionless quantities may be defined as follows

$x=\frac{x^{\prime}}{L}, \quad y=\frac{y^{\prime}}{L}, \quad t=\frac{t^{\prime} U_{r}}{L}$.

$u=\frac{u^{\prime}}{U_{r}}, \quad l=\frac{\iota^{\prime}}{U_{r}}, \quad T=\frac{T^{\prime}}{\Delta T^{\prime}}, \quad$ Bo $=\frac{g \rho L^{2}}{\sigma}$.

$\mathrm{Re}=\frac{U_{r} L}{\nu}, \quad \mathrm{Ma}=\frac{U_{r} L}{k}, \quad \mathrm{Gr}=\frac{g \beta \Delta T^{\prime} L^{3}}{v^{2}}$,

where the prime expresses dimensional quantities. for example, $\left(u^{\prime}, l^{\prime}, 0\right)$ is the dimensional velocity vector; $\sigma, \beta, \nu, k, \partial \sigma / \partial T$, and $g$ are, respectively, the surface tension coefficient, thermal expansion coefficient, kinematic viscosity, thermal conductivity coefficient, differentiation of surface-tension with respect to temperature, and the earth's gravitational acceleration. The non-dimensional parameter Bo is the static Bond number, Gr the Grashof number, Re the Reynolds number based on the typical velocity $U_{\mathrm{r}}$, and the Prandtl number $\operatorname{Pr}=\nu / k$. Marangoni number, Reynolds number and Prandtl number are connected by the relation $\mathrm{Ma}=\mathrm{Re} \mathrm{Pr}$.

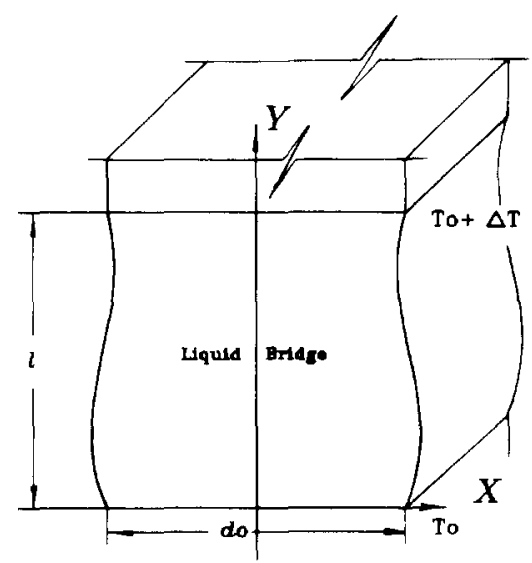

Fig. 1. Schematic diagram of 1wo-dimensional liquid bridge. 
We introduce the stream-function $\psi$ and vorticity $\omega$ in non-dimensional Cartesian coordinates $(x, y, z)$ as follows:

$u=\frac{\partial \psi}{\partial y}, \quad v=-\frac{\partial \psi}{\partial x}, \quad w=0$,

$\omega=\frac{\partial \ell^{\prime}}{\partial x}-\frac{\partial u}{\partial y}$.

Then, the dimensionless equations for $\psi$ and $\omega$ may be written as

$$
\begin{gathered}
\frac{\partial^{2} \psi}{\partial x^{2}}+\frac{\partial^{2} \psi}{\partial y^{2}}=-\omega, \\
\operatorname{Re}\left(\frac{\partial \omega}{\partial t}+\frac{\partial \psi}{\partial y} \frac{\partial \omega}{\partial x}-\frac{\partial \psi}{\partial x} \frac{\partial \omega}{\partial y}\right) \\
=\frac{\partial^{2} \omega}{\partial x^{2}}+\frac{\partial^{2} \omega}{\partial y^{2}}-\frac{\operatorname{Gr}}{\operatorname{Re}} \frac{\partial T}{\partial y}
\end{gathered}
$$

$\mathrm{Ma}\left(\frac{\partial T}{\partial t}+\frac{\partial \psi}{\partial y} \frac{\partial T}{\partial x}-\frac{\partial \psi}{\partial x} \frac{\partial T}{\partial y}\right)=\frac{\partial^{2} T}{\partial x^{2}}+\frac{\partial^{2} T}{\partial y^{2}}$.

The boundary conditions may be simplified as follows for the case of a fixed free surface:

$$
\begin{aligned}
& \psi(\theta, y, t)=\psi(1, y, t)=\psi[x, R(x), t]=0, \\
& \omega(0, y, t)=\omega(1, y, t)=\frac{2\left(\psi_{w}-\psi_{w}\right)}{d x^{2}}, \\
& \left.\omega\right|_{y=R(x)}=\frac{-4 R_{x} u_{x}}{\left(1-R_{x}^{2}\right)}+2 v_{x}+\frac{\left(1+R_{x}^{2}\right)}{\left(1-R_{x}^{2}\right)} \frac{\mathrm{d} T}{\mathrm{~d} S}, \\
& T(0, y, t)=0, T(1, y, t)=1, \\
& \frac{\frac{\partial T[x, R(x), t]}{\partial n}}{\partial n}=-R_{\mathrm{c}} T^{4},
\end{aligned}
$$

where $R_{\mathrm{c}}=\epsilon \mathrm{c} l \Delta T^{3} / k, \epsilon$ is the radiation coefficient and $c$ the Stefan-Boltzmann constant. $R_{\mathrm{c}}$ $=0$ is adopted as an approximation in the present calculation, because of the low temperature in the liquid bridge. The subscript $x$ denotes differentiation with respect to $x \cdot \psi_{w+1}$ and $\psi_{w}$ are respectively the stream function values at the distance $\Delta x$ from the wall and at the wall, and the equation of free surface is described as

$y=R(x)$
In Eq. (9), the vorticity at the free-surface is given by the equilibrium condition of tangential stresses. Then, the problem is to solve Eqs. (3)-(5) under boundary conditions (7)-(10) for specific parameter values.

\section{Numerical simulation}

In the present problem of time-dependent and two-dimensional thermocapillary oscillatory convection, the type of the basic equations is convection-diffusion. The hybrid method of fractional steps with the modified characteristic procedure for convection operator and the lumping finite element method for diffusion term was used in the present programme. The liquid bridge is divided into 544 triangular elements with 306 nodes. The linear interpolation and Galerkin technique were used for the discretion of differential equations. A steady solution of $\left(\psi_{0}, \omega_{0}, T_{0}\right)$ corresponding to $1^{\circ} \mathrm{C}$ of applied temperature difference between the top and bottom walls of the liquid bridge was adopted as the initial value.

For a fixed ratio width $d_{\min } / d_{0}$, the applied temperature difference was increased in a fixed heating rate from $1^{\circ} \mathrm{C}$ to a certain value. The calculation was repeated from initial state to an adopted higher applied temperature difference if the convection was steady; otherwise a lower value of $\Delta T$ was adopted if the convection was oscillatory. The calculation was repeated until the critical applied temperature difference was found. The marginal stability curve describing the dependence of critical applied temperature difference on the volume of the liquid bridge was obtained by changing the ratio width one by another in the calculation of the critical applied temperature.

The evolutionary processes of the maximum stream function and the surface temperature at $Y=0.02$ are shown in Figs. 2 and 3 for $d_{\min } / d_{0}=$ 0.5 and 0.9 , respectively. The flow and temperature fields become oscillatory when the applied temperature difference is larger than the critical value. The maximum stream function and surface temperature spectra are given in Fig. 4, which contains a single sharp peak and some harmonics. 

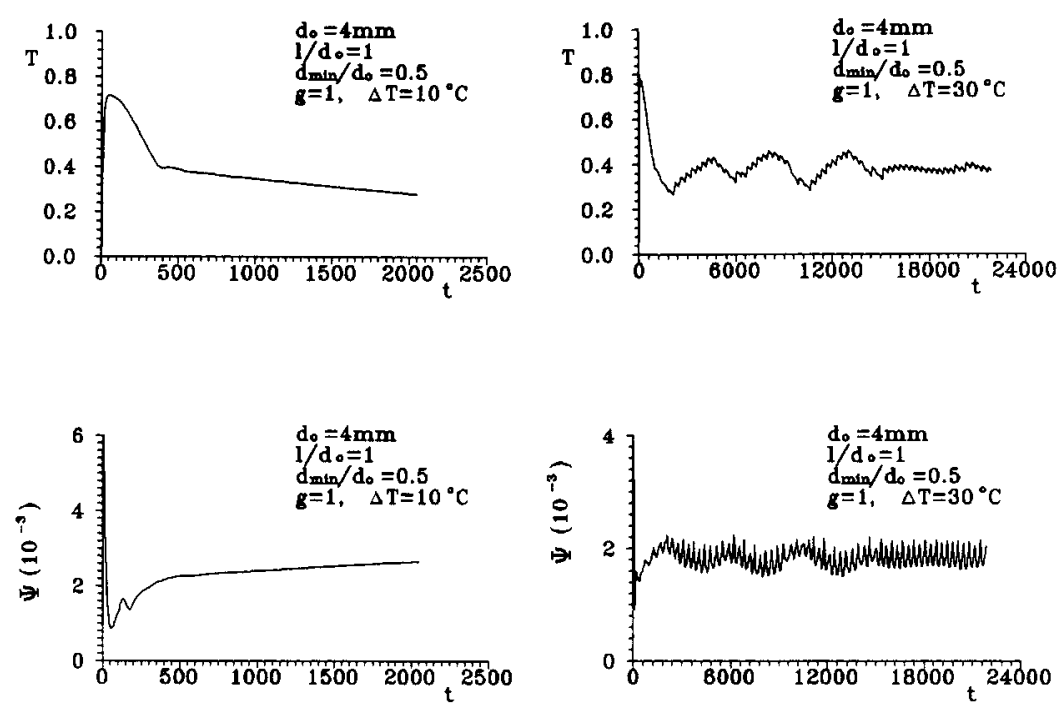

Fig. 2. Evolution of maximum stream functions and surface temperature at $Y=0.02$ for ratio width 0.5 .

It is clear that the main frequency is a little higher at $d_{\min } / d_{0}=0.5$ and $\Delta T=30^{\circ} \mathrm{C}$ than at ratio width $d_{\min } / d_{0}=0.9$ and $\Delta T=40^{\circ} \mathrm{C}$, i.e., a fatter liquid bridge has lower oscillatory frequency at the same applied temperature difference.

The patterns of time-dependent flow, described in the sequence of $1 / 8$ period of time, for liquid bridges with ratio width 0.5 and 0.9 , are shown in Figs. 5 and 6, respectively. These pat- terns are similar to the results observed in our experiments for the liquid bridge of half floating zone. The patterns in Fig. 5 have a slight asymmetry, and the relative difference is less than $3.3 \%$ for the maximum stream functions in left and right regions of the liquid bridge, and $0.81 \%$ for maximum relative temperature differance at the points of both free surfaces. However, in Fig. 6 each of the eight flow patterns is obviously asymmetric, and the maximum differences of
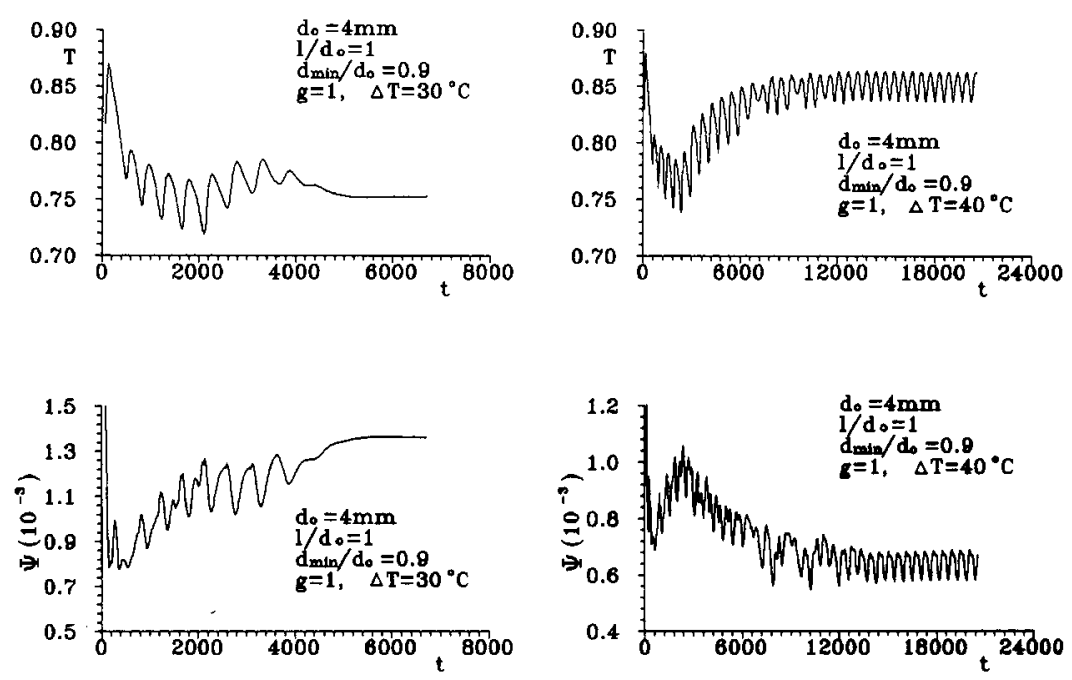

Fig. 3. Evolution of maximum stream function and surface temperature at $Y=0.02$ for ratio width 0.9 . 

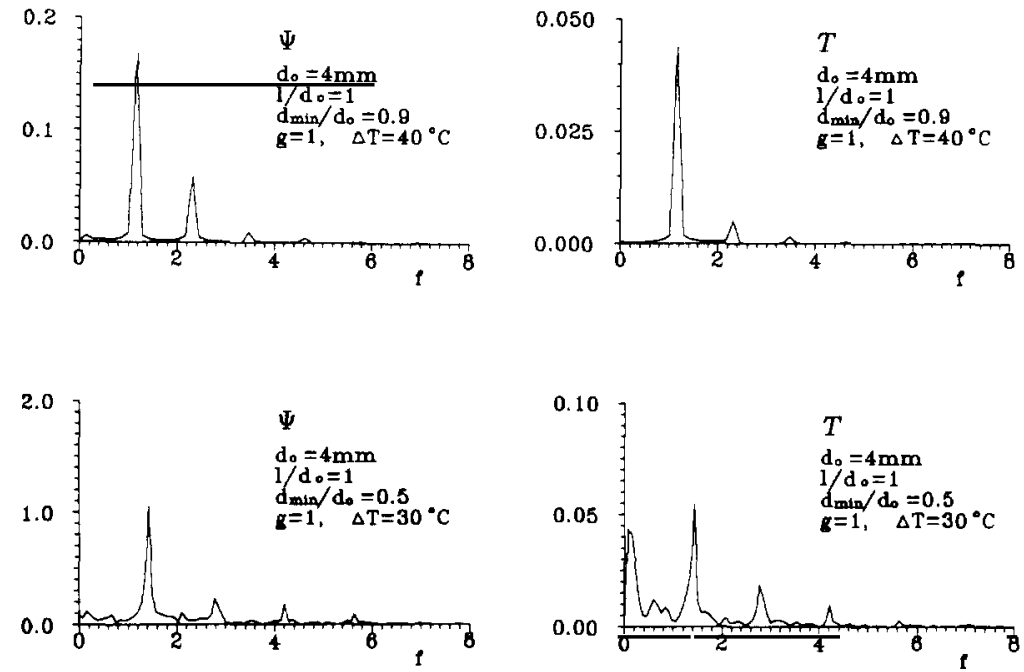

Fig. 4. Maximum stream function and surface temperature spectra.
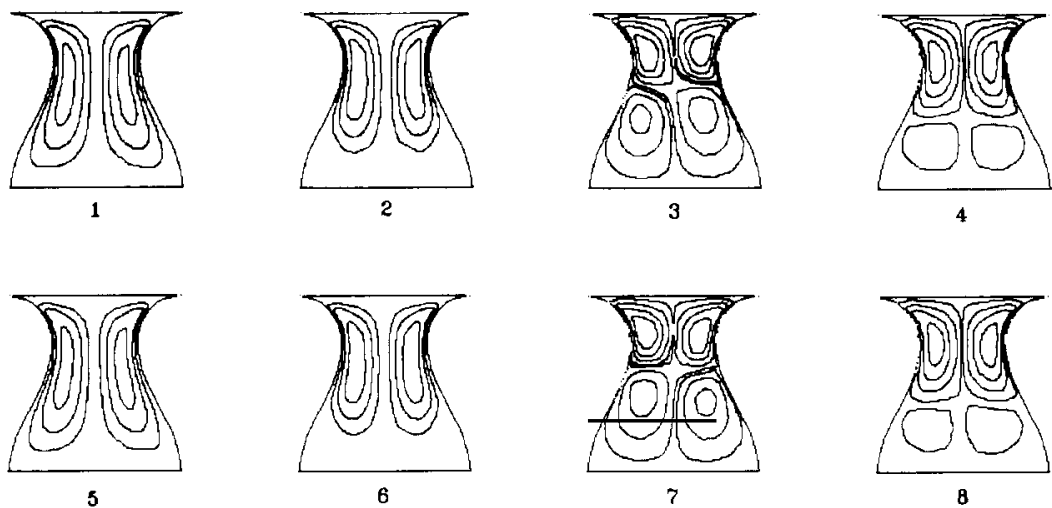

Fig. 5. Flow patterns in a period for $d_{\min } / d_{0}=0.5$, and $\Delta T=30^{\circ} \mathrm{C}$.
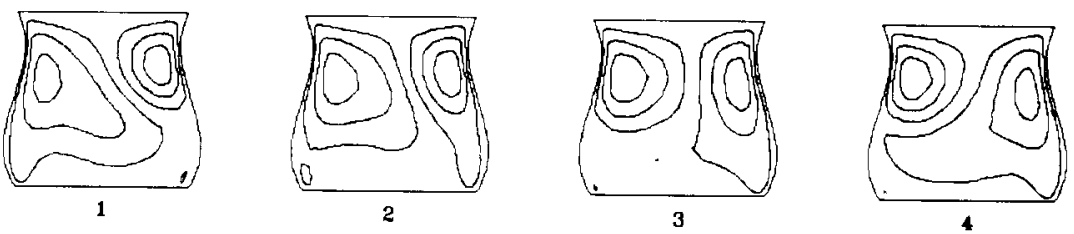

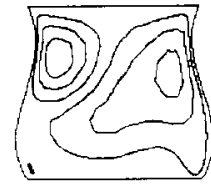

5

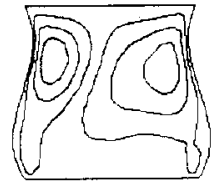

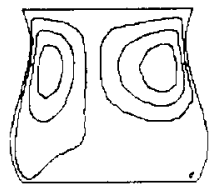

7

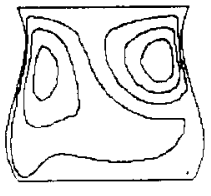

8

Fig. 6. Flow patterns in a period for $d_{\min } / d_{0}=0.9$, and $\Delta T=40^{\circ} \mathrm{C}$. 
stream functions in left and right regions of the liquid bridge and maximum temperature difference at both free surfaces are $15.76 \%$ and $3.5 \%$, respectively. It is obvious that the larger the ratio width, the more asymmetric the flow field and the temperature distribution.

The S-type surface temperature distribution can be observed obviously in Fig. 7. In our calculation, this kind of temperature distribution is always connected with the oscillatory thermocapillary convection.

The computational results show that the marginal curve between critical temperature difference and ratio width of the liquid bridge is divided into two branches from about $d_{\min } / d_{0}=$ 0.86 as shown in Fig. 8. There is a gap near $d_{\min } / d_{0}=0.86$, the value of the applied temperature difference is much higher than, for example, $200^{\circ} \mathrm{C}$. This means that the convection is kept steady for these liquid bridges at such a higher applied temperature difference. This is consistent quantitatively with the experimental results, but the left part of the marginal curve is not enhanced with a decrease of minimum width. It is probably due to the difference between two-dimensional and three-dimensional model. It should be noted that the right part of the marginal curves is smoother than the left part, where the oscillatory feature appears in the region close to the gap.

It is observed in our calculation that the critical temperature difference is different for increasing or decreasing applied temperature difference processes. The critical temperature dif-

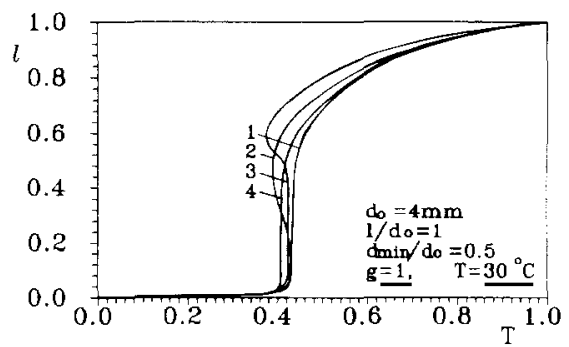

Fig. 7. Surface temperature distribution of the liquid bridge. Curves 1 to 4 are associated with 1 to 4 in Fig. 5 .

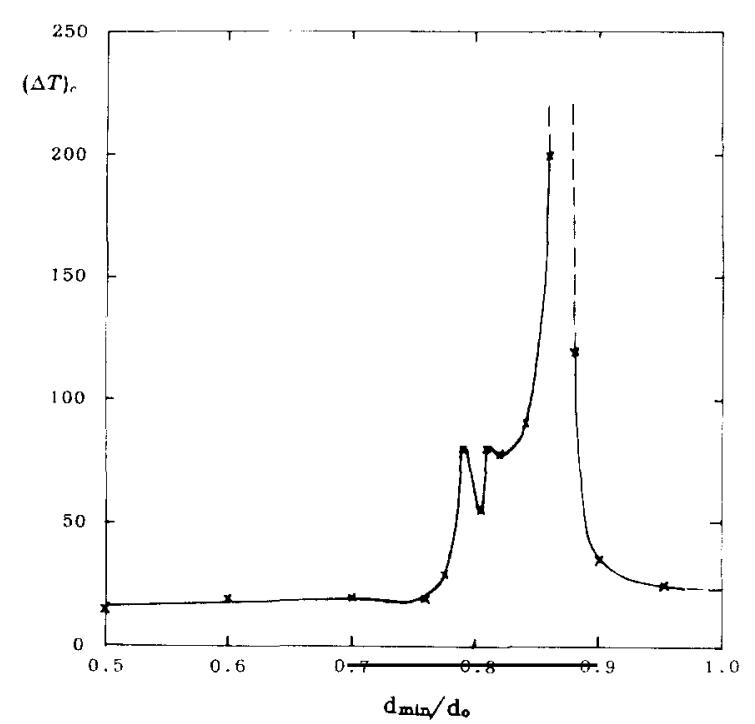

Fig. 8. Marginal stability curve associated with the critical applied temperature difference and the ratio width.

ference is higher in increasing temperature process than in decreasing temperature process.

\section{Conclusion}

The main experimental conclusion on the influence of the liquid bridge volume on the onset of oscillation presented in Paper $I$ is given in this paper by numerical simulation. The marginal stability curve relating the volume of the liquid bridge and the critical applied temperature difference was obtained numerically, and the main features of both experimental and numerical curves are quite similar. A gap region of liquid bridge volume with much larger value of critical temperature difference was also demonstrated numerically. The critical value of $(\Delta T)_{c}$ in the gap region was larger than $200^{\circ} \mathrm{C}$ in the present calculation. It means that the critical applied temperature difference in the gap region should be, at least, much larger than the usual value if it is finite. This agrees with the experimental conclusion that the critical value $(\Delta T)_{c}$ cannot be measured until the liquid bridge was broken. The larger value of the critical applied temperature difference means that the state is relatively stable 
on the one hand, and the larger gradient of marginal stability curve near the gap region and the oscillatory profile of the curve shows that the state is relatively unstable near the gap region. Anyway, the gap region of the liquid bridge volume is abnormal and should be understood in further research. The results of the present paper confirm the main conclusion given experimentally by Paper I about the dependence of critical value $(\Delta T)_{c}$ on the volume of the liquid bridge.

The numerical simulations were completed for a two-dimensional and unsteady model, and the simplification of the two-dimensional model introduced limitations in comparison with the real case of half floating zone. Furthermore, a fixed heating rate was used in the calculation process. It seems that these simplified assumptions should be improved in the next step; however, the essential features of the convection will not be changed by introducing these assumptions, especially for the mechanism studies. The results obtained in the present paper show that the numerical method designed for the thermocapillary convection was successful especially for the studies of the unsteady processes with free surface.

\section{References}

[1] Z.H. Cao, J.C. Xie, Z.M. Tang and W.R. Hu, Adv. Space Res. 11 (1979) 163.

[2] Z.H. Cao, J.C. Xie and W.R. Hu, Scientia Sinica 35 (1992) 725

[3] W.R. Hu, J.Z. Shu, R. Zhou and Z.M. Tang, J. Crystal Growth 142 (1994) 379.

[4] Z.M. Tang and W.R. Hu, Chinese Sci. Bull. 37 (1992) 1941.

[5] Z.M. Tang and W.R. Hu, Microgravity Quart. 1 (1992) 123. 